I was not aware that Dr. Sutton to move its head. There were a and Mr. Finch had tried acupunc- slight strabismus and rolling of the turation in anasarcous cases; but I am happy in adding my testimony to theirs, not doubting but that this simple operation will be found a most valuable auxiliary in the treatment of anasarca.

Lynn-Regis, Norfolk, Sept. 8, 1823,

Case of Hydrocephalus Chronicus, (Chronic Water of the Brain) in which Pressure proved most beneficial. By J. F. Barnard, Esq. Bedford, Member of the Royal College of Surgeons, London.


attending chronic hydrocephalus, now so frequent among children, I have been induced to submit to the readers of the Repository the following case :-

A child, about a year and a half old, was born, to all appearance, perfectly healthy, and continued so until six months old, when the head was first observed to increase in size. I did not see it until the disease was so far advanced that I almost entirely despaired of its terminating favourably. The head was exceedingly large, weighing, I should think, nearly as much as two-thirds of the rest of the body. The child lay in a state of stupor, and was unable in the least degree eye-balls, and almost constant startings of the muscles of the whole body, but more particularly of the face. The countenance had a cadaverous appearance, and the skin was of a yellowish colour. The eyes were sunk in their sockets, and enclosed in a dark ring. The flesh was flabby, and seemingly nanging on the muscles. The evacuations from the bowels were particularly unhealthy; sometimes green, sometimes blackish, but never of a healthy colour; nor indeed had they been healthy since half a year after its birth. The tongue was constantly covered with a thick white coat. When its head was moved it screamed, and seemed sensible of pain. I expected the child would survive but a few days. [I should say that it had been taking purgatives and mercurials, without benefit, for some time before I saw it.] I communicated my opinion to the parents, and told them the only chance I saw of saving their child was a plan which I shall describe, and which they readily assented to.

I had the head shaved perfectly clean : I then applied broad strips of adhesive plaster completely round the head from before back- 
ward, and cross strips from one larger than it ought to be. The side to the other, so as effectually flesh is firm, and the skin of a to support the parietes of the cra- healthy mottled hue. The water, nium. I ordered the whole head I have no doubt, will in time be to be kept constantly covered with absorbed, and he will completely linen dipped in cold water; and recover. I shall recommend the that the child should take no other bandage to be worn until the bones medicine than a liitle castor oil, should the buwels require it.Having thus decided on my practice, I patiently waited the result. Its good effects were evident in less than a week: the little patient could move its head much better; the strabismus squinting had disappeared; the secretions from the bowels were more healthy; and the startings of the muscles were less frequent. He had not screamed on rolling or moving the head since the bandage was applied. In a fortnight the size of the head had evidently lessened; the child was more lively, and began to take notice of the persons around it; the secretions from the bowels were perfectly healthy and evacuated regularly; the tongue nearly clean, and the skin of a natural colour ; and the countenance more composed and animated. It is now two months since the bandage was first applied; and the child is in every respect bealthy, except that the head sti 1 contains water, and is are fully united.

As this disease generally attacks more than one in a family, I have advised that the younger children should wear a small roller bandage on the head to support it during the time the bones are forming, and until they are perfectly united. If this were done more generally, as it used to be, I have no doubt it would prevent many of those dreadful cases of hydrocephalus we find in the present day. It is allowed by medical men that the disease is more frequent now than formerly; and to what greater cause can we ascribe it, than to the omitting of the application of that lind of bandage which was usually employed to protect the head of the infant from injury, and to support its imperfectly ossified and yielding parietes.

That this child was greatly relieved by the manner of bandaging which I employed, and that alone, I firmly believe. In order to ascertain more accurately the efficacy of this practice, I ordered 
that the child stould take no me- very cold, tongue parched, and dicine,-not even the castor oil, corered with a thick brown fur; unless it was absolutely requisite. pulse very quick (130), and wiry. It was necessary to give it small He had vomited frequently, and doses of the oil, but not oftener had passed several small slimy than once or twice a week, and stools. Let blood to the extent of that in the very ontset of the six ounces. Leeches to the templan. Its principal food during ples; warm bath, and friction with the clire was eggs and milk; I a coarse cloth or flannel. Take also allowed it a very small quan- four grains of calomel immeditity of wine, as a stimulant to the stomach.

Bedford, July 23d, 1823.

Case in which Hydatids were discharged in considerable quantities from the Intestincs. By Mr.T.M. Greenuow, Surgeon, Newcastle.

Master Sillkirk, aged 9, went to school on the morning of in May $2 \mathrm{~d}$, in his usual state of health. At $11 o^{\prime}$ clock, $a$. m. he was taken home ill, haring been saddenly seized with acute pain in the head and abdomen, accompanied with riolent shivering. I was requested to visit him at 9 o'clock the same evening. $\mathrm{He}$ then complained of severe pain in both temples, great pain and tenderness on pressure of the abdomen, impatience of light, eyes glassy, and wild in their expression, delirium, subsultus tendinum. His countenance was exceedingly oppressed, the surface generally ately, and a draught, with infusion of sennce and Epsom salts, in the morning. The bleeding produced sickness, and was followed by some remission of pain in the abdomen and temples.

May $3 d, 5$ o'clock, a. m.-Had been easier, and slept in the early part of the night, but had lately become worse. Much pain both in the head and abdomen; great restlessness and delirium. He had passed both urine and feces unconsciously. Pulse very quick and sharp; surface still very cold. Let blood to the extent of four ounces. To be sponged with warm vinegar and water, afterwards repeating the friction, and applying heat to the feet and hands. The stools were still scanty and slimy, and he had vomited the draught. Take four grains of calomel immediately, and, in one hour afterwards; half an ounce of castor oil.-10 a'clock, a.m. Head and abdomen have 
been easy since the last bleeding. calomel as last ordered, and in the Delirium continues, and he is very evening give an injection.-Evendrowsy. The castor oil was re-ing. Is somewhat better. Stools jected. Stools of the same cha- rather more plentiful. Pulse more racter, and still passed insensibly. distinct. Has slept in the course Pulse very quick, and small; of the day, and has retained some coldness of the surface continues. chicken broth. Less coldness of Apply a blister to the head, and the surface. Repeat the calomel. take three grains of calomel Repeat the bath, \&c. every four hours.-40'clock, p. $m$. No material change. Has vomited every thing except the calomel. No change in the nature of the stools; but he has used the pot since the morning. An efferyescing draught to be taken occasionally.-10 o'clock, $p . m$. The pulse is now a mere futter at the wrist, scarcely to be felt, and cannot be counted. No pain, but excessive restlessness and delirium; frequent hiccough ; stools as before; countenance greatly oppressed ; and the surface everywhere pale and cold Repeat the warm bath and friction.

May 4th, Morning.-Remains much in the same state. Says he has no pain. Stools as before. Take four grains calomel every four hours._Evening. No change. Repeat the bath and friction.

May 5th.-Stools of the same description. Pulse rather more distinct. The blister, which was applied on the third, remains on, but has not risen. Repeat the
May 6th, morning.-A great improvement has taken place. Large quantities of hydatids have been discharged from the bowels, accompanied with much mucus. All his bad symptoms have vanished. No pain; no delirium; tongue cleaner and moister. Skin comfortably warm and moist. Pulse soft, and not exceeding 100. The romiting has ceased, and he has had some refreshing sleep. More hydatids were discharged in the course of the day. The month became affected with the calomel, which was now omitted, 74 grains having been taken in three days.

From this time his convalescence has been progressive, the bowels being kept open with a mixture of magnesia, taken with lemon juice. That the remarkable matter discharged from the intestines of this patient was the cause of this very acute attack, cannot, I think, be doubted; especially when it is considered how all the unfavourable symptoms dis. 
appeared at the same instant of me on the $3 d$ and following days) its remoral from the system. How long it might have existed there, it would not be easy to determine; but its formation must probably have been going on for a very cousiderable length of time. For the last year he had been subject to occasional disordered action of the abdominal viscera, attended with griping, and local tenderness in the region of the liver. During the winter, an attack of this kind had been overcome by repeated doses of calomel and saline purges; and for several weeks he appeared in perfect health.

The hydatids discharged, in this case, were of various sizes and different colours. Some were transparent and light brown; others opaque and pale green, very nearly resembling boiled gooseberries, and of about the same dimensions. Others were much darker ; and the mucus which accompanied them was also of a dark green colour, probably from the effects of the calomel. They were unaccompanied by any feces, the whole being entirely without odour. I should, perhaps, have felt diffident in declaring this matter to consist of hydatids, notwithstanding its strongly marked character, had not the opinion of Dr. Ramsay (who had visited this patient with entirely coincided with my own respecting it.

This case appears to me curious and interesting, both on account of the train of alarming symptoms which were induced, and of the cause by which they appear to have been occasioned. When I was first called to this patient, I was impressed with a conviction that inflammatory action was going on in the abdomen, and that great determination existed to the head. In these circumstances, I thought it right to bleed, although the general state of the system rendered it necessary to do this with great caution; and though the quantity of blood drawn was small, the relief obtained was considerable; and after the second small bleeding, the pain both in the head and abdomen was permanently relieved. It will readily be supposed that little hopes of his recovery was entertained; and, indeed, on the evening of the 3d, I must confess I considered him actually in a dying state. The warm bath and friction 1 believe to have been the means of saving him at that time. It is remarkable, that, after a cold stage of pyrexia, which lasted more than three days, when at length (on the discharge of the offending matter from the intes- 
tines) reaction took place, it should the will directs the nervous stimuhave been so moderate and lus to the action of a certain set healthy. The incalculable rapi- of the roluntary muscles, at the dity and extreme oppression of the same time checking the action of all pulse at once subsided, and a moderate, soft, and equable action of the vascular system was established.-Edinburgh Medical and Surgical Journal.

Newcastle, May 24th, 1828.

On Obstruction of Blood in the Lungs.

WE shall now examine how far the obstruction of the blood in the lungs, from a deficiency of pure atmospherical air, is adequate to explain the cause of some of the vital phenomena observed in health and disease. All physiologists agree in admitting the direct dependence of the nervous system upon that of the sanguiferous; therefore the nervous system must lose its energy, in proportion as the circulation is diminished or impeded. As the nervous influence is the stimuius which excites the voluntary muscular system into action, it follows, that the latter must be enervated in proportion to the diminution of the energy of its stimulus. We often observe prostration of strength arising from a very short continuance of anxious exertion. $I$ allude to that exhaustion which comes on when the others. For example, two passionate lads fighting, in a few rounds (withont any serious injury, or a long continuance of unusual exertion), will fairly come to a stand, breathless and exhausted, with a sense of anxiety about the præcordia. To recruit themselves, they will "set to," as it is very emphatically called by the fancy, "bellows mending;" and, in a short time, the uneasiness about the heart will wear off, the strength will be recovered, and the contest will be renewed again with vigour. How are we to account for this rapid exhaustion, and equally rapid restoration of strength? Why, the muscles of respiration being semi-voluntary, are under the control of the will; when the will directs its whole attention to the action of any particular set of muscles, the function of respiration is checked as long as the uneasy sensation, from the accumulation of blood in the pulmonary artery, can be endured. The effect of this partial stagnation of the circulation is a diminution of the nervous stimulus to excite the muscular system into action. The more intent the will is in 
directing the nervous stimulus to any particular set of the voluntary muscles, the less sensible will the system be to the uneasy sensation arising from the accumulation of blood in the pulmonary artery; consequently, the sooner will there be a diminution of nervous energy to stimulate the muscular system. To relieve the exhaustion of strength, and the anxiety about the region of the heart, the system demands an additional supply of pure air to unload the pulmonary artery. As this congestion is removed, the unpleasant sensations vanish; and as soon as the system is renovated with the vital stream, it is invigorated, and the individual recovers his wonted strength. This view of the subject teaches every pugilist a valuable lesson, that is, never to interrupt his breathing unnecessarily, or, in other words, not to exhaust his strength by too anxiously watching his adversary's motions. It is well known, that a passionate pugilist exhausts himself much sooner than a cool one; and the reason is, that the passionate pugilist, from his great anxiety, is less sensible to the uneasy sensation occasioned by the accumulation of blood in the pulmonary artery, therefore he interrupts his breatbing for a longer period than the cool one; consequently, he exhausts himself sooner.

We shall next attempt to explain the cause of atn occurrence which often happens when we are in the full enjoyment of our health, but which is oftener a concomitant of one of the most fatal of diseases; I allude to the rupture of the pulmonary artery. Hrmoptysis, in my opinion, is generally the effect of an accumulation of blood in the pulmonary artery, arising from a deficiency of pure atmospherical air in the lungs, to decarbonate the blood immediately on its being conveyed into that viscus.The deficiency may arise from an interruption of the action of the respiratory muscles; as, from the immoderate use of the vocal organs; or, from inspiring rarified and impure air ; or, from the overdistension of the stomach, limiting the action of the diaphragm. Public speakers, singers, and performers on wind-instruments, are too well known to be the frequent victims of hæmoptysis. The enthusiastic orator, stimulated by the interest of his subject, and prond of the approbation of his audience, endeavours, by every exertion, to make the greatest impression upon his hearers; by so doing, he inter. 
rupts his respiration, and occasions ! lungs are unable to supply the ina partial accumulation of blood in crease of blood immediately with the pulmonary artery. If this in- pure air, so as to enable it to proterruption is often repeated, the ceed onwards without delay; therepulmonary artery must become fore, a temporary accumulation more and more dilated, as well as takes place in the pulmonary ardebilitated, and at last hæemop- tery, which must irritate its extysis will succeed ; or, from habitual irritation, the foundation of a more insidious disease will be laid, -I mean tubercular consumption. If the last conclusion be correct, we can account for the frequency of tubercular consumption, in countries subject to sudden vicissitudes of the atmosphere. The consequence of sudden and frequent changes of temperature must be sudden and frequent floods of blcod, as it were, rushing into the lungs, especially into the lungs of those who have very delicate and highly sensible constitutions. The pulmonary arte- ries of open-chested persons easily accommodate themselves to those frequent torrents; as the blood, from the capacity of their lungs, is immediately exposed to the influence of the atmosphere, and undergoes the necessary change to admit it to proceed onwards without any delay. The pulmonary arteries of narrow-chested persons, on the contrary, soon feel the effects of a sudden increase in the circulating medium; for their

To enter into the detail of every phenomenon, which, in my opinion, arises from the obstruction of the blood in the lungs, would lead me beyond the limits of an article intended for a periodical publication. If my corollary, that the blood is obstructed in the lungs from a deficiency of pure air, be correct, then I have no doubt that, from the labour of others, a rapid advancement will be made in the pathology of all the diseases of the respiratory organs. If our theory be admitted, it will probably lead, in the treatment of these diseases, to a firmer reliance on the efficacy of certain medicines of acknowledged power in regulating the circulation; and it will render most of us less susceptible of being made the dupes of charlatanism. It will, in many cases, suggest to us a valuable prophylactic; and it will procure a more ready compliance to our advice from many who are predisposed to diseases of the respiratory organs, more especially 
from public speakers. Most men are prone to turn a deaf ear to an advice which is to ward off what they suppose to be an imaginary evil. But, if we can convince a person that the blood stagnates in his lungs every time he interrupts his respiration, by uttering long periods, and that that interruption endangers the rupture of a blood vessel, or that it engenders in his bosom one of the most unrelenting of diseases (tubercular consumption), surely, under such an impression, no one will be found so careless of his health and life, as not to pay some share of attention, during his professional duty, to the important function of respiration.

Now, I flatter myself, that the cause of the phenomenon that reserved the discovery of the circulation of the blood to modern times, and to the honour of our country, has been disclosed, and that no one for the future, however sceptical, will be able to urge the vacuity of the arteries after death, as an objection to the doctrine of our immortal Harvey.

How far temporary accumulations of blood in the pulmonary artery are a source of disease, $I$ leave to the decision of time. Yet I must say, that Dr. Traill's coinciding with my views on the subject, has made me not a little san- guine, that my pathological speculations are founded upon a substantial hasis; and I cannot refrain acknowledging, that $I$ am gratefully sensible of my obligations to Dr. Traill for his kindness during the above inquiry, as well as at all others times.-Dr. Williams' Edinburgh Medical and Surgical Journal.

Fatal Effects of Fear.-A man of colour, of middle age, rather above the common stature, robust, and apparently in good health, was received into the London Hospital, labouring under a moderate sized aneurism of the femoral artery. $\Lambda n$ operation was proposed to him, to which he readily assented: on entering the theatre, however, he fainted; some wine and water was given to him, which he distinctly swallowed, and the operation was proceeded in, the artery exposed, and the ligature applierl, but not tightened. During the operation, it was observed that no pulsation could be felt in the tumour, but this was accounted. for by the fainting. Before tightening the ligature, it was suggested by the operator to wait until the pulsation was re-established. Some increased attention was then paid, to arouse the dormant energies of the patient; and it was remarked, 
that the syncope had continued an most is generally, on this account, unusual time. After the attempts the worst. We have little expehad been some time persevered rience of oil in this country. in, a more attentive observation From the result of the few trials I proved that he was quite dead. All the usual resuscitative means were tried, but without effect. On dissection, both sides of the heart were found empty, and the lungs tinged with blood : no other particular appearance was observable.

\section{MEDICAL EXTRACTS.}

Bread is not the worse for being hard, provided it be properly masticated. All hard and tough animal food, particularly if it be salted, which adds to its hardness, is of difficult digestion. It seems to be from its hardness that smoked meat is oppressive. Hard and tough animal food cannot, by mastication, be reduced to the loose, pultacious form which hard bread assumes.

There are few things in conmon use so oppressive as butter. It appears to be more so than the fat of meat. The fat of mutton is less difficult of digestion than the fat of beef; and the fat of venison less so than either. The same may be said of the fat of turtle; but all kinds of fat are oppressive to a weak stomach; and that of which we are inclined to eat the tion of a secreting organ. This is olive oil, to a stomach accustomed to it is tess opprosive probably, than most kinds of fat.

Cheese is, in general, still more difficult of digestion than either butter or fat. With their oily nature, it combines the hardness and toughness of the dry and compressed curd, which is very diff. cult of minute division.-Wilson on Indigestion.

The purgative medicines which I have chiefly used in fever, are, calomel, calomel and jalap, compound powder of jalap, aloes, solutions of any of the mild neutral salts, infusions of senna, and sometimes the two last conjoined. -Hanitton on Purgatives.

It would have been fortunate if medical inquirers had always followed the progress of diseases step by step, and viewed them as a whole, from the first deviation from health to their termination. A contrary procedure has often betrayed them into confusion and error.-Ibid.

A disordered state of secretion, either as to quantity or quality, will be the natural effect of irrita- 